\title{
Peroxynitrite Is a Key Mediator of the Cardioprotection Afforded by Ischemic Postconditioning In Vivo
}

\author{
Jianhui Li ${ }^{1,2}$, Noureddine Loukili², Nathalie Rosenblatt-Velin ${ }^{3}$, Pal Pacher ${ }^{4}$, François Feihl', Bernard \\ Waeber $^{3}$, Lucas Liaudet ${ }^{2,3^{*}}$
}

1 Department of Hepatobiliary Surgery, the First Affiliated Hospital, Zhejiang University, College of Medicine, Hangzhou, China, 2 Department of Intensive Care Medicine and Burn Center, Lausanne University Hospital Medical Center, Lausanne, Switzerland, 3 Division of Clinical Pathophysiology, Department of Internal Medicine, Lausanne University Hospital Medical Center, Lausanne, Switzerland, 4 Laboratory of Physiologic Studies, National Institute on Alcohol Abuse and Alcoholism, National Institutes of Health, Bethesda, Maryland, United States of America

\begin{abstract}
Myocardial ischemic postconditioning (PosC) describes an acquired resistance to lethal ischemia-reperfusion (I/R) injury afforded by brief episodes of I/R applied immediately after the ischemic insult. Cardioprotection is conveyed by parallel signaling pathways converging to prevent mitochondria permeability transition. Recent observations indicated that PostC is associated with free radicals generation, including nitric oxide (NO) and superoxide $\left(\mathrm{O}_{2} \cdot{ }^{-}\right)$, and that cardioprotection is abrogated by antioxidants. Since NO. And $\mathrm{O}_{2} \cdot$ react to form peroxynitrite, we hypothesized that postC might trigger the formation of peroxyntrite to promote cardioprotection in vivo. Rats were exposed to 45 min of myocardial ischemia followed by $3 \mathrm{~h}$ reperfusion. PostC (3 cycles of 30 seconds ischemia/30 seconds reperfusion) was applied at the end of index ischemia. In a subgroup of rats, the peroxynitrite decomposition catalyst $5,10,15,20-$ tetrakis(4-sulphonatophenyl) porphyrinato iron (FeTPPS) was given intravenously (10 mg/kg-1) 5 minutes before PostC. Myocardial nitrotyrosine was determined as an index of peroxynitrite formation. Infarct size (colorimetric technique and plasma creatine kinase-CK-levels) and left ventricle (LV) function (micro-tip pressure transducer), were determined. A significant generation of 3-nitrotyrosine was detected just after the PostC manoeuvre. PostC resulted in a marked reduction of infarct size, CK release and LV systolic dysfunction. Treatment with FeTPPS before PostC abrogated the beneficial effects of PostC on myocardial infarct size and LV function. Thus, peroxynitrite formed in the myocardium during PostC induces cardioprotective mechanisms improving both structural and functional integrity of the left ventricle exposed to ischemia and reperfusion in vivo.
\end{abstract}

Citation: Li J, Loukili N, Rosenblatt-Velin N, Pacher P, Feihl F, et al. (2013) Peroxynitrite Is a Key Mediator of the Cardioprotection Afforded by Ischemic Postconditioning In Vivo. PLoS ONE 8(7): e70331. doi:10.1371/journal.pone.0070331

Received May 24, 2013; Accepted June 23, 2013; Published July 10, 2013

Copyright: @ $2013 \mathrm{Li}$ et al. This is an open-access article distributed under the terms of the Creative Commons Attribution License, which permits unrestricted use, distribution, and reproduction in any medium, provided the original author and source are credited.

Funding: This work was supported, in part, by a grant from the Swiss National Fund for Scientific Research (Nr 320000/118174) to Lucas Liaudet and grants from the National Natural Science Foundation of China (Nr 81000137), the Qianjiang Talent Program of Zhejiang Province, China (Nr2012R10045), and the Scientific Research program for the Returned Overseas Chinese Scholars, Ministry of Health, Zhejiang Province, China (Nr 491010-G51104) to Jianhui Li. The funders had no role in study design, data collection and analysis, decision to publish, or preparation of the manuscript.

Competing interests: The authors have declared that no competing interests exist.

*E-mail: lucas.liaudet@chuv.ch

\section{Introduction}

Acute myocardial infarction due to coronary artery occlusion is the major cause of chronic heart failure in developed countries. Although timely reperfusion is mandatory to rescue the ischemic tissue, it may result by itself in additional myocardial injury and dysfunction, a concept termed reperfusion injury [1]. The most powerful strategies known so far to limit ischemia-reperfusion injury involve various techniques of myocardial conditioning [2], which describes an acquired resistance to lethal ischemia/reperfusion provided by brief episodes of ischemia applied either before ischemia (preconditioning, IPC) or just before reperfusion (postconditioning, PostC) [2,3]. On a clinical viewpoint, the concept of PostC is particularly attractive, as it might be applied as a simple measure at the onset of therapeutic reperfusion, as reviewed recently $[4,5]$. The mechanisms underlying cardioprotection by PostC are only partially elucidated, and there seems to be many similarities with those involved in IPC [5]. The PostC manoeuvre induces the release of various autacoids, such as bradykinin, adenosine and opioids, triggering receptor-dependent activation of several parallel intracellular signalling cascades. These include the Reperfusion Injury Salvage Kinase (RISK) pathway, the Survival Activating Factor Enhancement (SAFE) pathway, protein kinase $\mathrm{G}$ and protein kinase $\mathrm{C}$, which ultimately confer cadioprotection by inhibiting the opening of the mitochondrial permeablity transition pore thereby preventing subsequent cell death [6-8]. 
Recent evidence has been obtained that redox-dependent mechanisms play an important contribution to the activation of these cardioprotective signals. Both IPC and PostC are indeed associated with the generation of free radicals, including nitric oxide (NO) and the superoxide radical $\left(\mathrm{O}_{2}{ }^{-}\right)$, and treatment with antioxidant compounds prior to the conditioning stimulus abrogate cardioprotection $[9,10]$. Importantly, $\mathrm{NO}$ and $\mathrm{O}_{2}$. spontaneously react, in a diffusion-controlled process, to yield the potent oxidant and nitrating species peroxynitrite [11], whose formation during IPC has been shown to be involved in its protective actions [12-14]. With respect to PostC, one study reported on an increased formation of peroxynitrite during a PostC procedure performed in an ex vivo rat heart preparation [15], but the potential role of peroxynitrite during in vivo PostC is presently unknown. Such information is critical, as it would indicate that peroxynitrite may act as a protective mediator under certain conditions, contrasting with its established cytotoxic role in the reperfused myocardium [14,16]. We therefore conducted the present study in an in vivo model of myocardial ischemia and reperfusion, in order to test the hypotheses that PostC fosters the generation of peroxynitrite in the heart to promote cardioprotection in this setting.

\section{Methods}

\section{Ethic statement}

The investigation conformed to the Guide for the Care and Use of Laboratory Animals published by U.S. National Institutes of Health. All animal work was conducted according to relevant national and international guidelines and was performed with the approval of the Local Institutional Animal Care and Use Committee (Service of Veterinary Affairs, State of Vaud, Switzerland, authorization Nr 1502.2).

\section{Animal experimental procedures}

Male Wistar rats (10 weeks old, weight $250-300 \mathrm{~g}$, total number $=70$ ) were used in this study. Anesthesia was induced by intraperitoneal pentobarbital (60 $\mathrm{mg} \mathrm{kg}^{-1}$.) and was maintained by subsequent doses of intraperitoneal pentobarbital $\left(10 \mathrm{mg} \mathrm{kg}^{-1}\right)$ according to monitoring of the depth of the anesthesia using the plantar reflex response. Anesthetized animals were then intubated, and mechanically ventilated $\left(\mathrm{FiO}_{2} 0.3 ; 65\right.$ strokes $\left.\mathrm{min}^{-1}, 8 \mathrm{~mL} \mathrm{~kg}^{-1}\right)$ with a Harvard 683 rodent respirator (Holliston, MA, USA). A polyethylene (PE50) catheter was inserted into the right jugular vein for drug administration (see below). In a subset of animals, a Millar micro-tip pressure transducer was inserted into the right carotid artery for hemodynamic studies, as detailed below. Core temperature was maintained at $37 \pm 0.5^{\circ} \mathrm{C}$ with a heating pad. At the end of the experimental procedures, animals were euthanized with an intravenous overdose of pentobarbital (100 $\left.\mathrm{mg} \mathrm{kg}^{-1}\right)$.

\section{Myocardial ischemia reperfusion}

Myocardial ischemia-reperfusion (MIR) was performed according to our previously published procedure $[16,17]$. Briefly, the heart was exposed via a left thoracotomy and the left anterior descending coronary artery (LAD) was occluded by a small piece of PE tubing applied against the LAD by a $6-0$ silk suture passed underneath the artery. After 45 minutes ischemia, reperfusion was allowed for 3 hours by relieving the PE tubing. For sham experiments, the animals underwent the same procedures with the exception that the LAD was not occluded.

\section{Measurements}

\section{Left ventricular hemodynamics}

Hemodynamic studies were performed in $n=5$ rats per group, using a micro-tip pressure catheter (SPR-671, 1.4 Fr; Millar Instruments Inc., Houston, TX), inserted into the left ventricle (LV) via the right carotid artery. Heart rate, LV end-diastolic (LVEDP) and end-systolic (LVESP) pressures were recorded, and the maximal and minimal rates of change of LV pressure ( $\mathrm{dP} / \mathrm{dt}$ max and $\mathrm{dP} / \mathrm{dt} \mathrm{min}$ ) were calculated as load-dependent indices of LV contractility and relaxation, using a PowerLab/4SP AD converter (A D Instruments, Oxfordshire, UK).

\section{Determination of Myocardial Infarct Size and plasma creatine kinase activity}

Area at risk (AAR) and infarct size were determined in $n=7$ rats/group (except from the sham group, without infarction), using the triphenyl tetrazolium chloride (TTC)-Evans blue technique, as previously described [16-18]. At the end of the experiments, heparinized whole blood was drawn from the inferior vena cava, centrifuged at $3000 \mathrm{rpm}$, and plasma was stored at $-80^{\circ} \mathrm{C}$. Plasma creatine kinase activity $(\mathrm{CK})$ and its myocardial $\mathrm{MB}$ fraction (CKMB) were measured as an indicator of myocardial necrosis, using a commercial kit (Sigma Chemicals, St Louis, MO, USA).

\section{SDS-PAGE and western immunoblot detection of 3- nitrotyrosine in the myocardium}

Groups of $n=5$ animals were killed after 45 minutes ischemia (or sham ischemia), just before reperfusion or immediately following PostC, and the activity of 3-nitrotyrosine (3-NT) was determined in myocardial samples from the ischemic LV as a marker of peroxynitrite generation, according to our previously published procedure [16]. Briefly, myocardial tissue was homogeneized (TrisHCl $10 \mathrm{mM}, \mathrm{NP} 40$ 0.5\%, NaCl $0.15 \mathrm{M}, \mathrm{Na}$ ${ }_{3} \mathrm{VO}_{4} 1 \mathrm{mM}$, NaF $10 \mathrm{mM}$, PMSF $1 \mathrm{mM}$, EDTA $1 \mathrm{mM}$, aprotinin $10 \mu \mathrm{g} / \mathrm{ml}$, leupeptin $10 \mu \mathrm{g} / \mathrm{ml}$, and pepstatin $1 \mu \mathrm{g} / \mathrm{ml}$ ), and proteins $(30 \mu \mathrm{g})$ were separated by SDS-PAGE, transferred to nitrocellulose membrane, and blocked for $1 \mathrm{~h}$ at room temperature with $5 \%$ nonfat dry milk in Tris-buffered saline with $0.1 \%$ Tween 20 . The membrane was incubated overnight at $4^{\circ} \mathrm{C}$ with a 1:1000 dilution of a mouse monoclonal antinitrotyrosine antibody (Upstate Biotechnology, Lake Placid, NY), followed by incubation with a horseradish peroxidaseconjugated secondary antibody at a 1:5,000 dilution for $1 \mathrm{~h}$. The immunoblot signal was visualized using enhanced chemiluminescence (ECL, Amersham Biosciences, Otelfingen, Switzerland) and quantified by densitometric analysis. The 


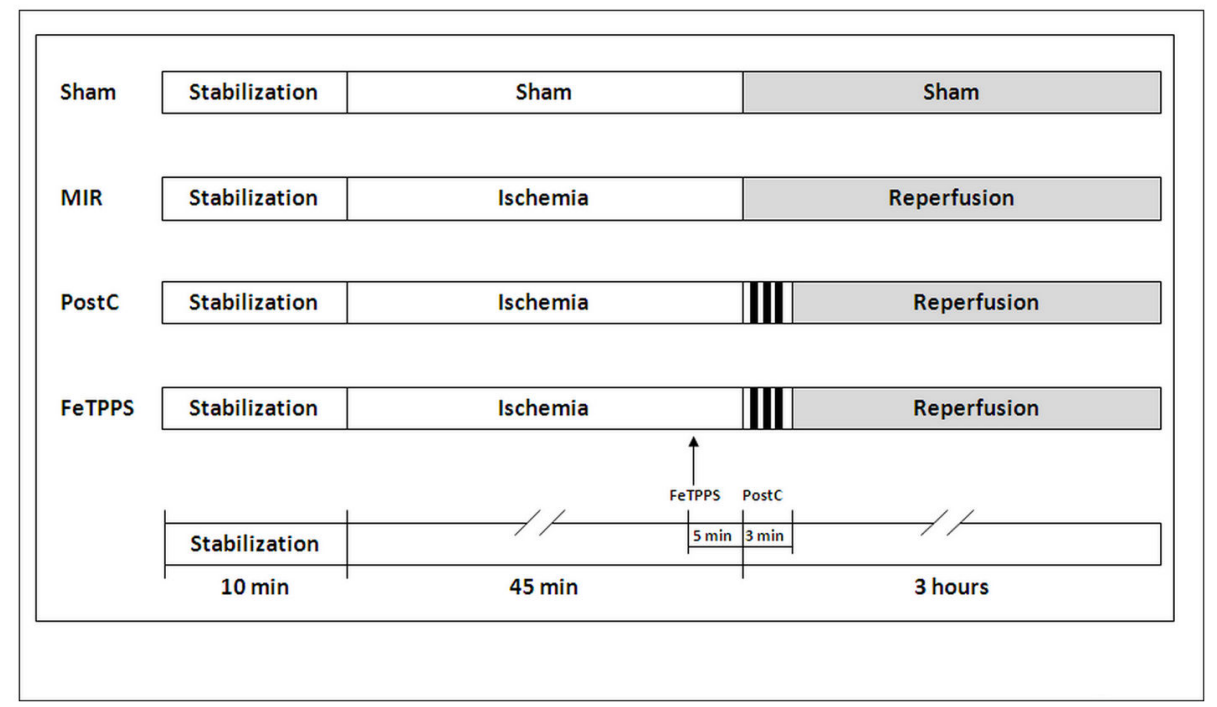

Figure 1. Experimental protocol (Figure 1). Four groups of rats were investigated, including Sham group, MIR group (45 minutes ischemia and $3 \mathrm{~h}$ reperfusion), PostC group (3 cycles of 30 seconds ischemia/reperfusion applied at the end of the 45 minutes ischemia, and preceding the $3 \mathrm{~h}$ reperfusion), and FeTPPS group (treatment with the peroxynitrite decomposition catalyst 5,10,15,20-tetrakis(4-sulphonatophenyl) porphyrinato iron (FeTPPS), $10 \mathrm{mg} / \mathrm{kg}$ intravenously 5 minutes before PostC [18]).

doi: 10.1371/journal.pone.0070331.g001

levels of tubulin were determined in parallel as a loading control.

\section{Statistical analysis}

All values are expressed as mean $\pm \mathrm{SD}$. Data were analyzed by one-way analysis of variance followed by the Dunnett's test for multiple comparisons. A $p<0.05$ was considered to be statistically significant.

\section{Results}

\section{Ischemic postconditioning induces 3-NT formation in the myocardium}

As illustrated in Figure 2, there was no increased signal for 3-NT following 45 minutes of ischemia. In contrast, a marked increase of the 3-NT was detected immediately after the end of the 3 cycles of PostC, indicating that the postC manoeuvre promoted the formation of significant amounts of peroxynitrite within the ischemic LV.

\section{The reduction of myocardial infarct size by PostC is blunted by FeTPPS}

The area at risk (AAR, the ischemic area, Figure 3A) was comparable among the three groups of animals. Infarct size, whether expressed as a percentage of the AAR (Figure 3B), or as a percentage of the total LV (Figure 3C), was markedly and significantly reduced by PostC, an effect significantly attenuated by FeTPPS. Figure 3D shows representative images of Evans blue-TTC staining of LV from the the three groups of rats. Furthermore, the large increase of plasma CK
(Figure 4A) and CKMB (Figure 4B) following MIR was significantly reduced by PostC, but this effect was abolished by FeTPPS.

\section{FeTPPS abolishes the benefit of PostC on post- ischemic myocardial systolic dysfunction, but not diastolic dysfunction}

As shown in Figure $5 \mathrm{~A}$, no significant alteration of heart rate was noted across the different experimental groups. When compared to sham animals, rats exposed to MIR disclosed a significant reduction of LVESP together with a significant drop of $\mathrm{dp} / \mathrm{dt} \max$ (Figure $5 \mathrm{~B}$ and $5 \mathrm{C}$ ), pointing to a marked decrease of LV contractility. The reduced contractility was significantly less pronounced following PostC, but this benefit was lost upon treatment with FeTPPS. Furthermore, ischemiareperfusion also resulted in a significant impairment of diastolic relaxation, as indicated by an increased LVEDP and a reduced $\mathrm{dp} / \mathrm{dt}$ min (Figure 5D and 5E). PostC suppressed the increase of LVEDP and tended to attenuate the decrease of $\mathrm{dp} / \mathrm{dt} \mathrm{min}$, albeit nonsignificantly $(p=0.08, t$ test). These effects were not significantly influenced by FeTPPS.

\section{Discussion}

The present investigation brings two important novel conclusions with respect to the mechanism of cardioprotection afforded by postconditioning in vivo. First, we report that a brief protocol of postconditioning is sufficient to foster the generation of significant amounts of peroxynitrite in the myocardium. Secondly, we show that the beneficial effects of postconditioning, both in terms of myocardial infarct size and cardiac systolic function, disappear when a peroxynitrite 


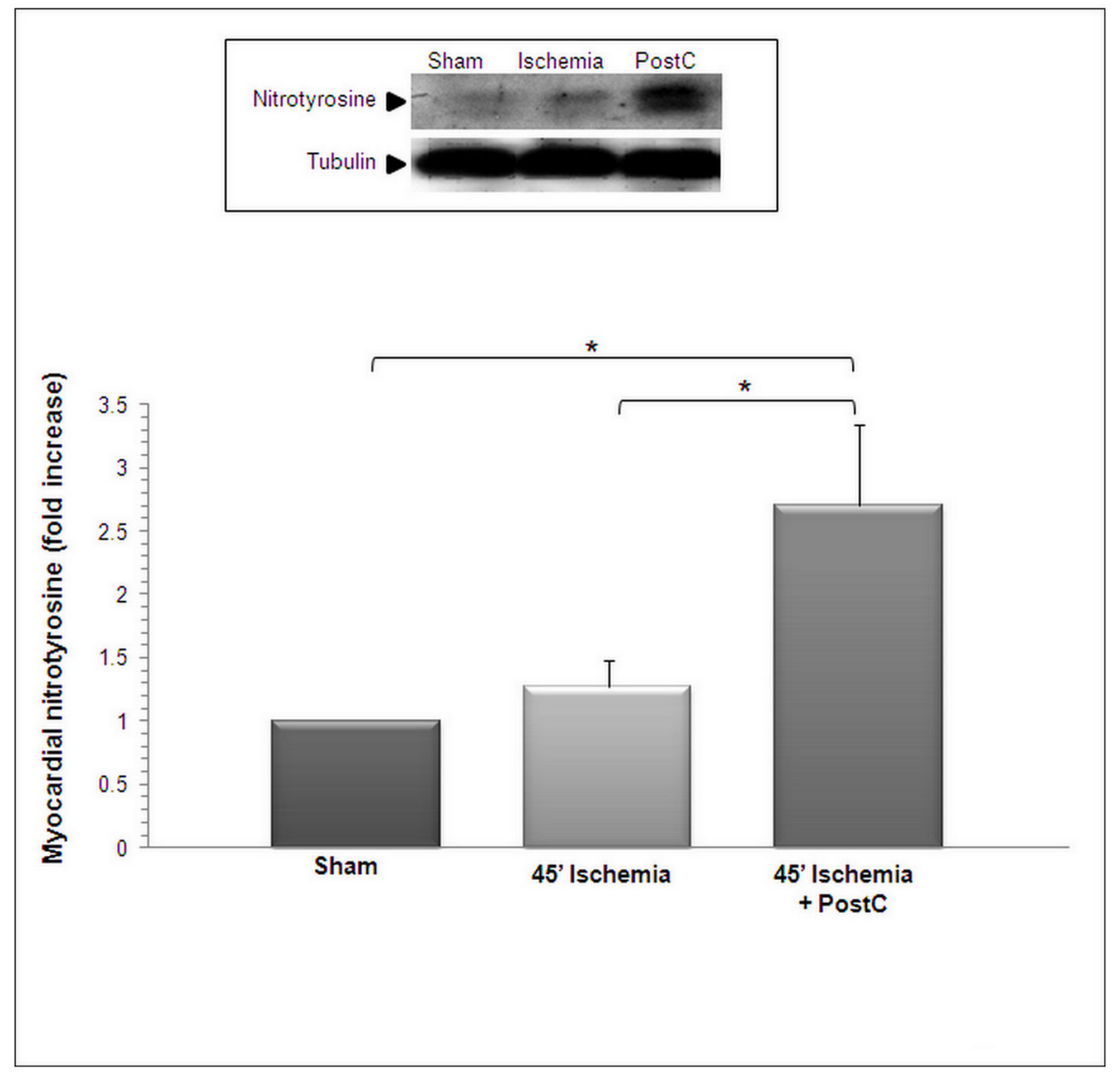

Figure 2. Postconditioning triggers 3-nitrotyrosine formation in the left ventricle. Nitrotyrosine was determined in the ischemic left ventricle obtained after 45 minutes sham ischemia, 45 minutes ischemia, or 45 minutes ischemia + PostC with 3 cycles of 30 seconds ischemia/reperfusion. Means $\pm S D$ of $n=5$ rats/condition. ${ }^{*} p<0.05$.

doi: 10.1371/journal.pone.0070331.g002

decomposition catalyst is given prior to postconditioning. Thus, our data demonstrate that peroxynitrite formation during postconditioning is a key mechanism underlying its cardioprotective actions in vivo.

Our protocol of postconditioning, established according to Zhao et al [3], allowed a significant reduction of myocardial infarct size, in agreement with previous investigators in a similar rat model [19]. Another important finding was the marked improvement of left ventricular systolic function, as shown by better preservation of LV systolic pressure and dp/dt max. Furthermore, PostC also improved diastolic function, indicated by reduced LV end-diastolic pressure and a blunted reduction of $\mathrm{dp} / \mathrm{dt} \mathrm{min}$, although the latter effect was only marginally significant $(p=0.08)$. While such functional improvement from postC has been previously reported in ex vivo models of myocardial ischemia [20] [21], our results indicate that PostC also ameliorates systolic and diastolic dysfunction in a relevant in vivo model of myocardial infarction.

Multiple identified mechanisms convey the cardioprotection of postC. Physiological mechanisms include maintenance of tissue acidosis and improved coronary endothelial function
[22]. Molecular mechanisms rely on the activation of several parallel signalling pathways -protein kinase G, the RISK and SAFE pathways, protein kinase C-, conferring cardioprotection through the inhibition of mitochondrial permeability transition pore (MPTP) opening, and the activation of mitochondrial KATP channel $[7,9,23]$. Limited evidence has also emerged that redox-based mechanisms might be involved in posconditioning [8]. Penna et al. showed that the antioxidant $\mathrm{N}$ acetylcysteine suppressed the benefit of PostC in an ex vivo rat model [24], whereas Tsutsumi et al. reported that the ROS scavenger 2-mercaptopropionyl glycine (MPG) suppressed the effects of PostC in an in vivo mouse model [25]. Furthermore, Lemoine et al. also showed that MPG abolished the effects of PostC on functional recovery of human cardiac muscle after hypoxia and reoxygenation in vitro [26]. Overall, these findings support the concept that the generation of oxidants during postC can represent an essential trigger of its cardioprotective effects. It has been proposed that such effects might depend on redox-mediated activation of protein kinase $\mathrm{C}$ and subsequent stimulation of adenosine-dependent signaling [6]. 


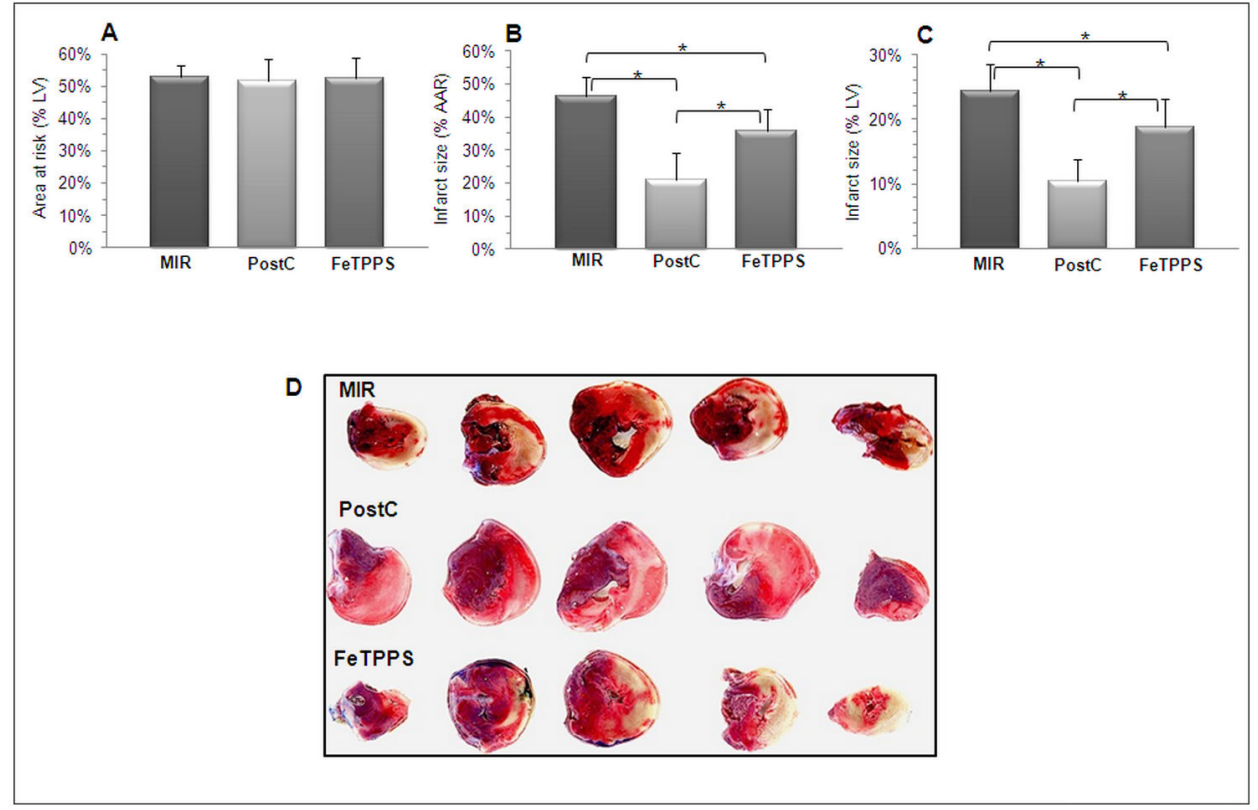

Figure 3. The reduction of infarct size by PostC is attenuated by FeTPPS. Rats exposed to myocardial ischemia ( $45 \mathrm{~min})$ and reperfusion (3h) were left untreated (MIR group) or were exposed to PostC, in the absence (PostC group) or in the presence of a 10 $\mathrm{mg} / \mathrm{kg}$ treatment with FeTPPS just before PostC (FeTPPS group). Area at risk (A) was comparable among the 3 groups of rats. Infarct size (B: \% AAR; C: \% left ventricle) was reduced by PostC, an effect suppressed by FeTPPS. D: Representative pictures of Evans blue-TTC staining of left ventricles from the the three groups of rats. Means $\pm S D$ of $n=7$ rats/group. * $p<0.05$. doi: 10.1371/journal.pone.0070331.g003

Our present findings provide additional support for a role of redox-based mechanisms in postC. First, we found that the postC manoeuvre elicited a significant generation of peroxynitrite in the myocardium, as evidenced by increased tyrosine nitration, and secondly, we found that treatment with FeTPPS just before PostC abolished the beneficial effects of the latter on infarct size and LV systolic dysfunction. Interestingly, FeTPPS did not alter the effect of PostC on diastolic dysfunction, suggesting that postC improves cardiac contractility and relaxation via distinct mechanisms, a hypothesis that should be explored in future investigations. With respect to tyrosine nitration, it is noteworthy that this posttranslational modification may occur independently from peroxynitrite, via myeloperoxydase-dependent catalysis in the presence of nitrite [11]. This mechanism is however highly unlikely in the conditions of our study, since the postC manoeuvre was too brief to elicit significant leukocyte accumulation within the myocardium. We speculate that the formation of peroxynitrite during PostC was promoted owing to the simultaneous generation of its 2 precursors in the postischemic and immediate reperfusion phase [14]. The primary source of $\mathrm{O}_{2} \cdot$ in this setting is the mitochondrion, which produces a burst of $\mathrm{O}_{2}$ - upon reoxygenation via the autooxidation of unstable semiquinones in the respiratory chain $[27,28]$. Regarding NO, its formation increases during ischemia, both through NOS-dependent synthesis and through the non enzymatic reduction of tissue nitrite in acidic $\mathrm{pH}$ [29].
Our results confirm and extend those of a previous study in an ex vivo model, showing that peroxynitrite played a role in the cardioprotection of PostC in isolated hearts obtained from normal, but not hyperlipidemic rats [15]. These findings may appear counterintuitive at first glance, in view of the numerous detrimental actions of peroxynitrite reported in the setting of myocardial ischemia-reperfusion injury and other heart diseases, and which include lipid peroxidation, DNA oxidative damage and activation of poly(ADP-ribose) polymerase (PARP), as well as the activation of matrix metalloproteinases, to name only a few $[30,31]$. However, it must be underscored that peroxynitrite does not only trigger direct cytotoxic effects, but it also promotes multiple indirect effects related to the modulation of an array of cell signaling pathways [32]. Such effects may depend either on an oxidative or a nitrative type of chemistry elicited by peroxynitrite [11], whose respective roles were not evaluated in the present study. With respect to PostC, it is here particularly noticeable that peroxynitrite has been shown to be a potent activator of ERK [33] and protein kinase $C$ [34] in the heart, two crucial kinases involved in cardioprotection by PostC. Therefore, although our study was not designed to determine the pathways downstream of peroxynitrite-dependent protection, we may speculate on such activation of ERK and PKC as a plausible mechanism. A distinct possibility may be linked with the activation of the enzyme PARP by peroxynitrite, which has been reported to be critical for the cardioprotection elicited by preconditioning [35]. Whether a similar scenario applies to the PostC paradigm 


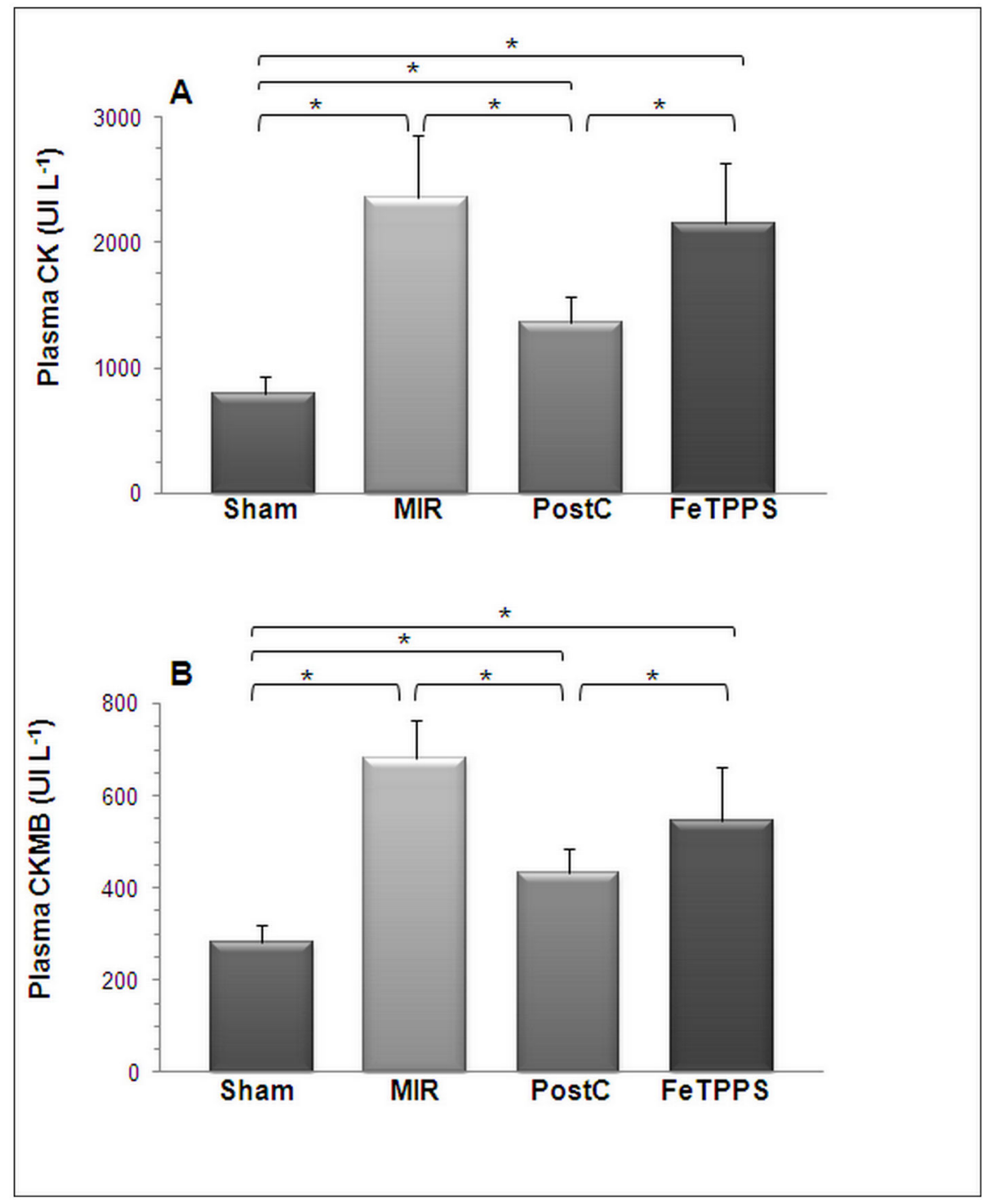

Figure 4. FeTPPS suppresses the effects of PostC on plasma CK and CKMB activity. Myocardial ischemia-reperfusion (MIR group) induced a large increase of plasma CK (A) and CKMB (B) activities. These increases were largely reduced by postconditioning (PostC group), but this effect was eliminated by FeTPPS treatment before PostC (FeTPPS group). Means \pm SD of $\mathrm{n}=7-9$ rats/group. ${ }^{*} \mathrm{p}<0.05$.

doi: 10.1371/journal.pone.0070331.g004

remains, however, to be established. Finally, a benefit from peroxynitrite formation during PostC might also partly depend on some preservation of the coronary endothelium and reduction of leukocyte-endothelial interactions, as supported from studies by Lefer and Nossuli et al. [36,37].

A limitation of our study is the lack of direct demonstration of peroxynitrite scavenging by FeTPPS. Although we $[18,38]$ and others [39] previously showed that FeTPPS efficiently catalyze the decomposition of peroxynitrite, this compound may also partly react with oxidant species distinct from peroxynitrite, such as hydrogen peroxide [40]. Therefore, we cannot formally rule out that some of the observed actions of FeTPPS in the present work might have been related to effects distinct from peroxynitrite decomposition.

In conclusion, our present results indicate that peroxynitrite is a proximal mediator of cardioprotection during PostC in vivo. These beneficial effects contrast with the established cytotoxicity of peroxynitrite in the reperfused myocardium, which suggests that anti-oxidant strategies for therapeutic purposes might produce variable effects on the infarcted heart, depending on the time of intervention. This should be considered in future studies evaluating antioxidants for the treatment of myocardial infarction. 


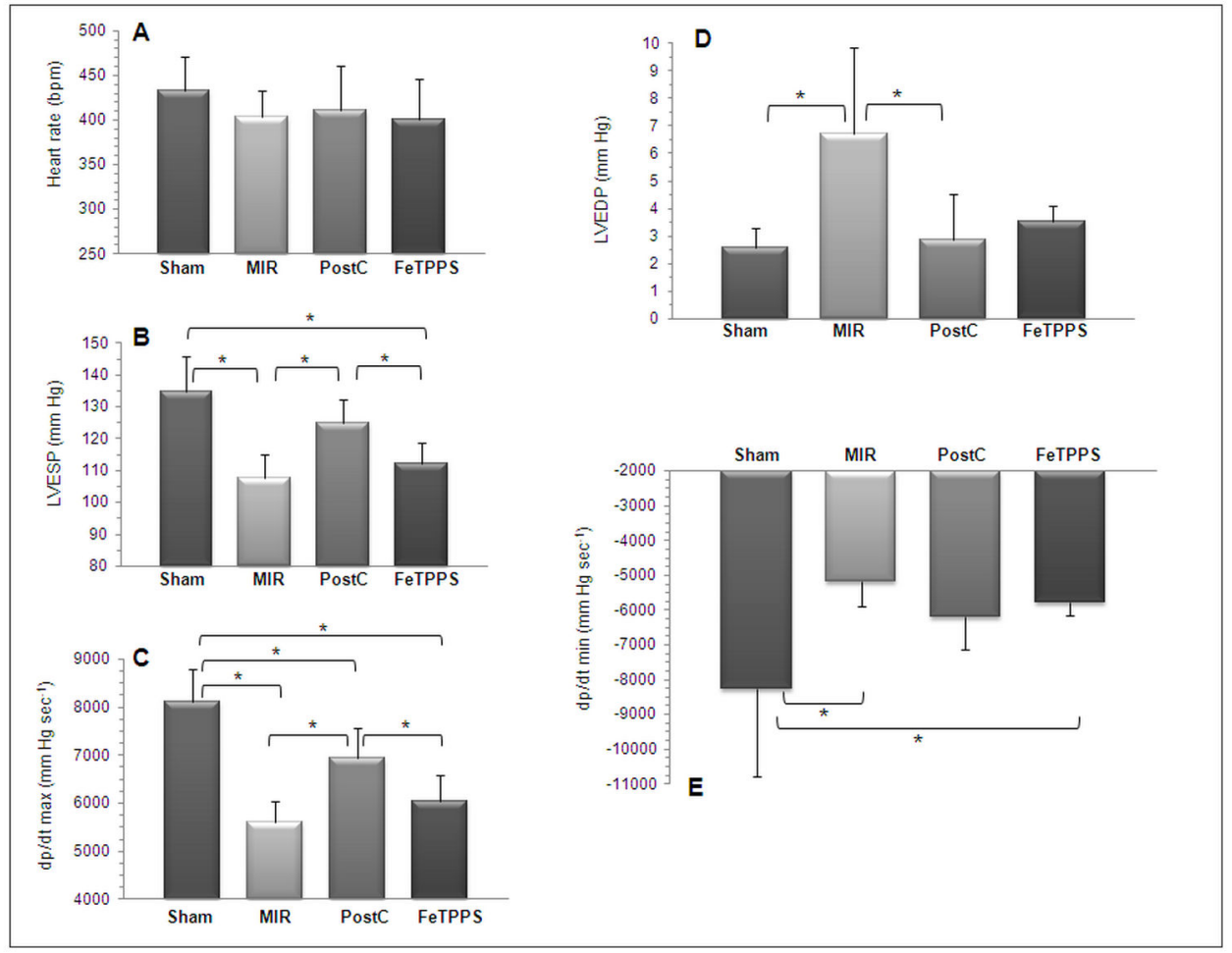

Figure 5. FeTPPS suppresses the beneficial effects of PostC on left ventricular contractility, but does not influence diastolic function. At the end of the observation period, heart rate was comparable among the 4 groups of rats (A). Myocardial ischemia and reperfusion (MIR group) induced a decrease of left ventricular end-systolic pressure (LVESP, B) and dp/dt max (C), which was attenuated by postconditioning (PostC group). FeTPPS suppressed the effect of PostC (FeTTPS group). Myocardial ischemia and reperfusion also induced a significant increase of left ventricular end-diastolic pressure (D: LVEDP) and a significant decrease of $\mathrm{dp} / \mathrm{dt} \min (\mathrm{E})$. PostC suppressed the increase of LVEDP, and tended to limit the reduction of $\mathrm{dp} / \mathrm{dt}$ min, and these effects were not significantly influenced by FeTPPS. Means \pm SD of $n=5$ rats/group. * $p<0.05$.

doi: 10.1371/journal.pone.0070331.g005

\section{Author Contributions}

Conceived and designed the experiments: LL NRV PP. Performed the experiments: JL NL NRV. Analyzed the data: LL

\section{References}

1. Yellon DM, Hausenloy DJ (2007) Myocardial reperfusion injury. N Engl $J$ Med 357: 1121-1135. doi:10.1056/NEJMra071667. PubMed: 17855673.

2. Sanada S, Komuro I, Kitakaze M (2011) Pathophysiology of myocardial reperfusion injury: preconditioning, postconditioning, and translational aspects of protective measures. Am J Physiol Heart Circ Physiol 301: H1723-H1741. doi:10.1152/ajpheart.00553.2011. PubMed: 21856909.

3. Zhao ZQ, Corvera JS, Halkos ME, Kerendi F, Wang NP et al. (2003) Inhibition of myocardial injury by ischemic postconditioning during reperfusion: comparison with ischemic preconditioning. Am J Physiol Heart Circ Physiol 285: H579-H588. PubMed: 12860564.

4. Mewton N, Bochaton T, Ovize M (2013) Postconditioning the heart of ST-elevation myocardial infarction patients. Circ J 77: 1123-1130. doi: 10.1253/circj.CJ-13-0385. PubMed: 23575305.

5. Ovize M, Baxter GF, Di Lisa F, Ferdinandy P, Garcia-Dorado D et al. (2010) Postconditioning and protection from reperfusion injury: where do we stand? Position paper from the Working Group of Cellular Biology of the Heart of the European Society of Cardiology. Cardiovasc Res 87: 406-423. doi:10.1093/cvr/cvq129. PubMed: 20448097.
PP FF BW. Contributed reagents/materials/analysis tools: LL FF BW. Wrote the manuscript: LL PP FF.

6. Cohen MV, Downey JM (2011) Ischemic postconditioning: from receptor to end-effector. Antioxid Redox Signal 14: 821-831. doi: 10.1089/ars.2010.3318. PubMed: 20518705.

7. Heusch G (2013) Cardioprotection: chances and challenges of its translation to the clinic. Lancet 381: 166-175. doi:10.1016/ S0140-6736(12)60916-7. PubMed: 23095318.

8. Penna C, Perrelli MG, Pagliaro P (2013) Mitochondrial pathways, permeability transition pore, and redox signaling in cardioprotection: therapeutic implications. Antioxid Redox Signal 18: 556-599. doi: 10.1089/ars.2011.4459. PubMed: 22668069.

9. Pagliaro P, Moro F, Tullio F, Perrelli MG, Penna C (2011) Cardioprotective pathways during reperfusion: focus on redox signaling and other modalities of cell signaling. Antioxid Redox Signal 14: 833-850. doi:10.1089/ars.2010.3245. PubMed: 20649460.

10. Penna C, Mancardi D, Rastaldo R, Pagliaro P (2009) Cardioprotection: a radical view Free radicals in pre and postconditioning. Biochim Biophys Acta 1787: 781-793. doi:10.1016/j.bbabio.2009.02.008. PubMed: 19248760

11. Pacher $P$, Beckman JS, Liaudet $L$ (2007) Nitric oxide and peroxynitrite in health and disease. Physiol Rev 87: 315-424. doi:10.1152/physrev. 00029.2006. PubMed: 17237348. 
12. Altug S, Demiryürek AT, Kane KA, Kanzik I (2000) Evidence for the involvement of peroxynitrite in ischaemic preconditioning in rat isolated hearts. $\mathrm{Br} \mathrm{J}$ Pharmacol 130: 125-131. doi:10.1038/sj.bjp.0703280. PubMed: 10781007.

13. Altup S, Demiryürek AT, Ak D, Tungel M, Kanzik I (2001) Contribution of peroxynitrite to the beneficial effects of preconditioning on ischaemia-induced arrhythmias in rat isolated hearts. Eur $\mathrm{J}$ Pharmacol 415: 239-246. doi:10.1016/S0014-2999(01)00843-3. PubMed: 11275005.

14. Ferdinandy P, Schulz R (2003) Nitric oxide, superoxide, and peroxynitrite in myocardial ischaemia-reperfusion injury and preconditioning. $\mathrm{Br} \mathrm{J}$ Pharmacol 138: 532-543. doi:10.1038/sj.bjp. 0705080 . PubMed: 12598407.

15. Kupai K, Csonka C, Fekete V, Odendaal L, van Rooyen J et al. (2009) Cholesterol diet-induced hyperlipidemia impairs the cardioprotective effect of postconditioning: role of peroxynitrite. Am J Physiol Heart Circ Physiol 297: H1729-H1735. doi:10.1152/ajpheart.00484.2009. PubMed: 19734363.

16. Levrand S, Vannay-Bouchiche C, Pesse B, Pacher P, Feihl F et al. (2006) Peroxynitrite is a major trigger of cardiomyocyte apoptosis in vitro and in vivo. Free Radic Biol Med 41: 886-895. doi:10.1016/ j. freeradbiomed.2006.04.034. PubMed: 16934671.

17. Liaudet L, Szabo E, Timashpolsky L, Virág L, Cziráki A et al. (2001) Suppression of poly (ADP-ribose) polymerase activation by 3 aminobenzamide in a rat model of myocardial infarction: long-term morphological and functional consequences. $\mathrm{Br} \mathrm{J}$ Pharmacol 133: 1424-1430. doi:10.1038/sj.bjp.0704185. PubMed: 11498530.

18. Loukili N, Rosenblatt-Velin N, Li J, Clerc S, Pacher P et al. (2011) Peroxynitrite induces HMGB1 release by cardiac cells in vitro and HMGB1 upregulation in the infarcted myocardium in vivo. Cardiovasc Res 89: 586-594. doi:10.1093/cvr/cvq373. PubMed: 21113057.

19. Manintveld OC, Te Lintel Hekkert $M$, van den Bos EJ, Suurenbroek $\mathrm{GM}$, Dekkers DH et al. (2007) Cardiac effects of postconditioning depend critically on the duration of index ischemia. Am J Physiol Heart Circ Physiol 292: H1551-H1560. PubMed: 17122197.

20. Penna C, Tullio F, Merlino A, Moro F, Raimondo S et al. (2009) Postconditioning cardioprotection against infarct size and post-ischemic systolic dysfunction is influenced by gender. Basic Res Cardiol 104: 390-402. doi:10.1007/s00395-008-0762-8. PubMed: 19030912

21. Fantinelli JC, Mosca SM (2007) Comparative effects of ischemic pre and postconditioning on ischemia-reperfusion injury in spontaneously hypertensive rats (SHR). Mol Cell Biochem 296: 45-51. doi:10.1007/ s11010-006-9296-2. PubMed: 16933149.

22. Shi W, Vinten-Johansen J (2012) Endogenous cardioprotection by ischaemic postconditioning and remote conditioning. Cardiovasc Res 94: 206-216. doi:10.1093/cvr/cvs088. PubMed: 22323534.

23. Ovize M, Baxter GF, Di Lisa F, Ferdinandy P, Garcia-Dorado D et al. (2010) Postconditioning and protection from reperfusion injury: where do we stand? Position paper from the Working Group of Cellular Biology of the Heart of the European Society of Cardiology. Cardiovasc Res 87: 406-423. doi:10.1093/cvr/cvq129. PubMed: 20448097.

24. Penna C, Rastaldo R, Mancardi D, Raimondo S, Cappello S et al. (2006) Post-conditioning induced cardioprotection requires signaling through a redox-sensitive mechanism, mitochondrial ATP-sensitive $\mathrm{K}+$ channel and protein kinase C activation. Basic Res Cardiol 101: 180-189. doi:10.1007/s00395-006-0584-5. PubMed: 16450075

25. Tsutsumi YM, Yokoyama T, Horikawa Y, Roth DM, Patel HH (2007) Reactive oxygen species trigger ischemic and pharmacological postconditioning: in vivo and in vitro characterization. Life Sci 81: 1223-1227. doi:10.1016/j.Ifs.2007.08.031. PubMed: 17915258.

26. Lemoine S, Buléon C, Rouet R, Ivascau C, Babatasi G et al. (2010) Bradykinin and adenosine receptors mediate desflurane induced postconditioning in human myocardium: role of reactive oxygen species. BMC Anesthesiol 10: 12. doi:10.1186/1471-2253-10-12. PubMed: 20670410.

27. Figueira TR, Barros MH, Camargo AA, Castilho RF, Ferreira JC et al. (2013) Mitochondria as a Source of Reactive Oxygen and Nitrogen Species: From Molecular Mechanisms to Human Health. Antioxid Redox Signal 18: 2029-2074. doi:10.1089/ars.2012.4729. PubMed: 23244576.

28. González-Flecha B, Cutrin JC, Boveris A (1993) Time course and mechanism of oxidative stress and tissue damage in rat liver subjected to in vivo ischemia-reperfusion. J Clin Invest 91: 456-464. doi:10.1172/ JCl116223. PubMed: 8432855.

29. Zweier JL, Wang P, Samouilov A, Kuppusamy P (1995) Enzymeindependent formation of nitric oxide in biological tissues. Nat Med 1 : 804-809. doi:10.1038/nm0895-804. PubMed: 7585184.

30. Pacher P, Liaudet L, Bai P, Mabley JG, Kaminski PM et al. (2003) Potent metalloporphyrin peroxynitrite decomposition catalyst protects against the development of doxorubicin-induced cardiac dysfunction. Circulation 107: 896-904. doi:10.1161/01.CIR.0000048192.52098.DD. PubMed: 12591762

31. Pacher P, Schulz R, Liaudet L, Szabó C (2005) Nitrosative stress and pharmacological modulation of heart failure. Trends Pharmacol Sci 26: 302-310. doi:10.1016/j.tips.2005.04.003. PubMed: 15925705.

32. Liaudet L, Vassalli G, Pacher P (2009) Role of peroxynitrite in the redox regulation of cell signal transduction pathways. Front Biosci 14: 4809-4814. PubMed: 19273391

33. Pesse B, Levrand S, Feihl F, Waeber B, Gavillet B et al. (2005) Peroxynitrite activates ERK via Raf-1 and MEK, independently from EGF receptor and p21Ras in H9C2 cardiomyocytes. J Mol Cell Cardiol 38: 765-775. doi:10.1016/j.yjmcc.2005.02.020. PubMed: 15850570.

34. Balafanova Z, Bolli R, Zhang J, Zheng Y, Pass JM et al. (2002) Nitric oxide (NO) induces nitration of protein kinase Cepsilon (PKCepsilon), facilitating PKCepsilon translocation via enhanced PKCepsilon -RACK2 interactions: a novel mechanism of no-triggered activation of PKCepsilon. J Biol Chem 277: 15021-15027. doi:10.1074/ jbc.M112451200. PubMed: 11839754.

35. Liaudet L, Yang Z, Al-Affar EB, Szabó C (2001) Myocardial ischemic preconditioning in rodents is dependent on poly (ADP-ribose) synthetase. Mol Med 7: 406-417. PubMed: 11474134.

36. Nossuli TO, Hayward R, Scalia R, Lefer AM (1997) Peroxynitrite reduces myocardial infarct size and preserves coronary endothelium after ischemia and reperfusion in cats. Circulation 96: 2317-2324. doi: 10.1161/01.CIR.96.7.2317. PubMed: 9337206

37. Lefer DJ, Scalia R, Campbell B, Nossuli T, Hayward R et al. (1997) Peroxynitrite inhibits leukocyte-endothelial cell interactions and protects against ischemia-reperfusion injury in rats. J Clin Invest 99: 684-691. doi:10.1172/JCl119212. PubMed: 9045871.

38. Levrand S, Pacher P, Pesse B, Rolli J, Feihl F et al. (2007) Homocysteine induces cell death in $\mathrm{H} 9 \mathrm{C} 2$ cardiomyocytes through the generation of peroxynitrite. Biochem Biophys Res Commun 359: 445-450. doi:10.1016/j.bbrc.2007.05.147. PubMed: 17544363.

39. Duplain H, Sartori C, Dessen P, Jayet PY, Schwab M et al. (2008) Stimulation of peroxynitrite catalysis improves insulin sensitivity in high fat diet-fed mice. J Physiol 586: 4011-4016. doi:10.1113/jphysiol. 2008.154302. PubMed: 18591189

40. Klassen SS, Rabkin SW (2009) The metalloporphyrin FeTPPS but not by cyclosporin $A$ antagonizes the interaction of peroxynitrate and hydrogen peroxide on cardiomyocyte cell death. Naunyn Schmiedebergs Arch Pharmacol 379: 149-161. doi:10.1007/ s00210-008-0342-3. PubMed: 18773197. 